\title{
Supressão do ground roll utilizando deconvolução de Wiener-Levinson no domínio tau-p
}

Fabiane S. Serpa, Michelângelo G. Silva, Milton J. Porsani - CPGG/UFBA, Brasil.

Copyright 2006, SBGf - Sociedade Brasileira de Geofísica

Este texto foi preparado para a apresentação no II Simpósio de Geofísica da Sociedade Brasileira de Geofísica, Natal, 21-23 de setembro de 2006. Seu conteúdo foi revisado pela Comissão Tecno-científica do II SR-SBGf mas não necessariamente representa a opinião da SGBf ou de seus associados. É proibida a reprodução total ou parcial deste materia para propósitos comerciais sem prévia autorização da SBGf.

\section{Resumo}

No processamento de dados sísmicos terrestres, a remoção do ground roll representa um sério problema. Esse evento ocorre na forma de um cone de ruído coerente, de alta amplitude e baixa velocidade de grupo, que se superpõe ao sismograma mascarando as reflexões de interesse.

Neste trabalho, a filtragem do ground roll foi conduzida através da deconvolução de Wiener-Levinson, aplicada a sismogramas transformados para o domínio das ondas planas (tau-p). O método foi testado sobre dados sísmicos da Bacia do Tacutu e os resultados obtidos demonstram que a abordagem utilizada, para atenuação do ground roll, é mais eficaz que o método de filtragem FK convencional, permitindo gerar uma imagem sísmica final de melhor qualidade, exibindo melhor resolução temporal e melhor continuidade lateral das reflexões.

\section{Introdução}

Em geral, os dados sísmicos de reflexão são sempre contaminados com ruídos. Nos dados sísmicos de levantamentos terrestres, um exemplo de tal ruído é o ground roll, associado a ondas superficiais do tipo Rayleigh, que mascaram as reflexões originadas nas camadas profundas. O ground roll propaga-se nas camadas superficiais de baixa velocidade, correspondentes ao manto de intemperismo, e demarcam os registros sísmicos (sismogramas) com eventos coerentes lineares, de baixo conteúdo de freqüência, alta amplitude e forte inclinação, indicando baixa velocidade de propagação (entre $100 \mathrm{~m} / \mathrm{s}$ a $1000 \mathrm{~m} / \mathrm{s}$ ). Normalmente, possui sua energia mais concentrada nas baixas freqüências em torno de $10 \mathrm{~Hz}$. A figura (1) ilustra o ground roll.

Este ruído é altamente dispersivo no domínio do tempo-offset e apresenta caráter preditivo (coerente) traço-a-traço. Sua velocidade de fase é limitada por $V_{\min }$ e $V_{\max }$ e, conseqüentemente forma um cone, o qual é chamado de cone de ruído. Sua alta amplitude e baixa freqüência interfere de forma destrutiva, mascarando e dificultando a identificação dos refletores de interesse. A presença desse ruído de baixa freqüência e de alta amplitude é um grande problema a se tentar contornar na geofísica de exploração. Muitos métodos de filtragens têm sido propostos para atenuar este ruído tais como, filtro $f-k$, passa-banda entre outros. Estes métodos conseguem atenuar o ground roll porém também interferem no conteúdo de freqüência das reflexões e atenuam também as reflexões. É pois importante o desenvolvimento de métodos de filtragem que atenuem o ground roll preservando as reflexões subjacentes ao cone de ruído.

Neste trabalho, utilizamos o método de deconvolução de Wiener-Levinson (WL), bastante utilizado no processamento de dados sísmicos, aplicando-o sobre sismogramas transformados para o domínio das ondas planas (tau-p). $O$ método de WL é bastante conhecido pela sua eficácia em aumentar o conteúdo de alta freqüência dos dados. Esse método utiliza um filtro preditivo que é obtido a partir da autocorrelação dos traços sísmicos. A deconvolução de WL é normalmente aplicada sobre os sismogramas com o objetivo de aumentar a resolução temporal das seções sísmicas, através da compressão do pulso sísmico.

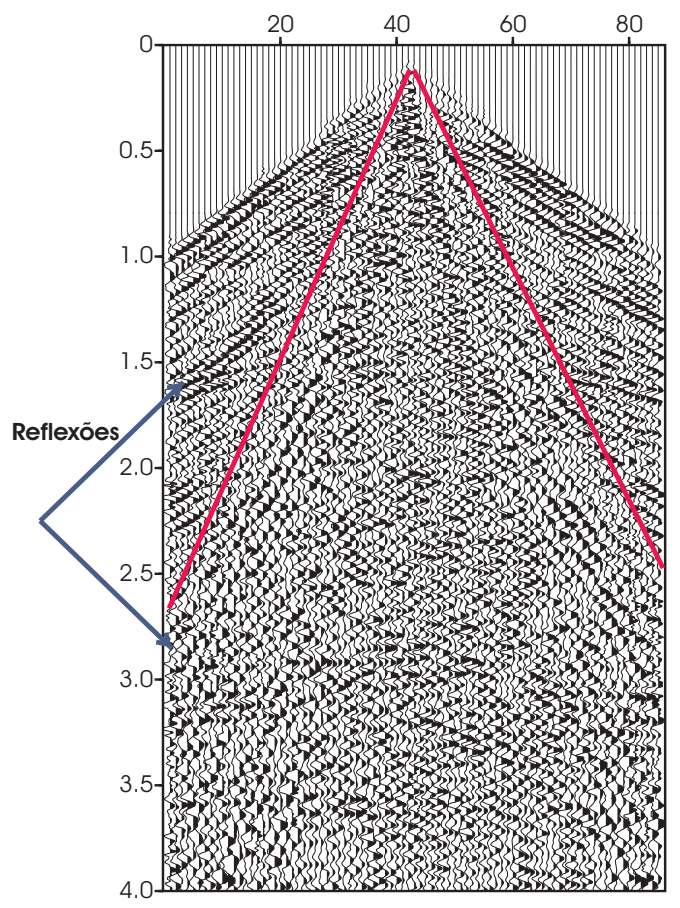

Figura 1: Sismograma de tiro comum da linha sísmica RL50-90. Em destaque o cone de ruído (ground roll). 


\section{Metodologia}

O seguinte procedimento foi utilizado para filtragem do ground roll

1. Aplicação da transformada tau-p aos sismogramas;

2. Obtenção do filtro de WL e deconvolução dos dados no domínio tau-p;

3. Aplicação da transformada inversa tau-p, e

4. Continuidade do fluxo normal de processamento para obtenção da seção empilhada final.

A seguir apresentamos um resumo do método de transformada tau-p e do método de deconvolução de WL.

\section{Transformada no domínio tau-p}

A decomposição em ondas planas (transformada tau-p ou transformada Slant-Stack) converte uma seção sísmica registrada $t-x$ para tempo de interseção $\tau$ e parâmetro de raio $p$.

Para se transformar dados em função do tempo duplo de reflexão e do afastamento da fonte aos receptores, para o domínio $\tau-p$, partimos da equação:

$$
F(\tau, p)=\int f(\tau+p x, x) d x
$$

onde, $f(\tau+p x, x)=f(t, x)$ representa o sismograma obtido com uma fonte pontual.

Esta equação representa uma reta que é tangente à curva do tempo de reflexão no ponto (t-x) cuja inclinação tem valor $p$ e o tempo de interseção $t=\tau$.

As equações de tempo de percurso no domínio tau-p podem ser obtidas considerando-se o modelo bidimensional com uma camada de espessura $Z_{0}$ e velocidade de propagação $V=$ constante. A curva tempo de chegada do evento refletido na interface $R$ é expressa por:

$$
t^{2}=t_{o}^{2}+\frac{x^{2}}{V^{2}}
$$

Esta equação descreve uma hipérbole (figura2) e representa o equacionamento dos tempos de percurso da onda sísmica no domínio $t-x$ onde,

$$
t_{o}=\frac{2 . Z_{o}}{V}
$$

representa o tempo de percurso para a incidência normal à interface.

Para se encontrar a equação da curva que representa o tempo de chegada do evento refletido em função do parâmetro do raio $p$ deve-se igualar as equações da reta $t=\tau+p x$ com a da hipérbole, equação 2 , bem como suas derivadas de primeira ordem. Assim obtém-se,

$$
\left(\frac{\tau}{t_{o}}\right)^{2}+(p V)^{2}=1 .
$$

Essa equação corresponde a uma elipse no plano $\tau-p$ (figura3), cuja interseção com o eixo vertical e horizontal é determinada pelo tempo duplo de percurso normal, $t_{o}$, e pelo inverso da velocidade da camada, $1 / V$, respectivamente.

Explicitando $\tau$ obtém-se,

$$
\tau=t_{o}\left(1-(p V)^{2}\right)^{1 / 2} .
$$

$\mathrm{Na}$ verdade, a curva de tempo de percurso $t(x)$ não é observada, é inferida a partir da seção $t-x$ registrada e é esta função que é mapeada no domínio $\tau-p$. A parametrização de tempo de interseção ou demora vertical $\tau$ e parâmentro de raio $p$ oferece vantagens não apenas em termos de apresentação visual dos dados no plano tau $-p$, mas também na formulação matemática exigida para obter a resposta do meio. Em contraste à fórmula aproximada usualmente empregada no domínio $t-x$, Diebold e Stoffa (1981) deduziram as expressões exatas para a curva de recepções de uma onda no domínio tau - $p$, para as geometrias comumente empregadas no processamento dos dados, estas equações podem ser aplicadas a dados com qualquer afastamento fonte-receptor. Existe, portanto, neste domínio, a possibilidade de comparação dos dados com uma resposta teórica exata.

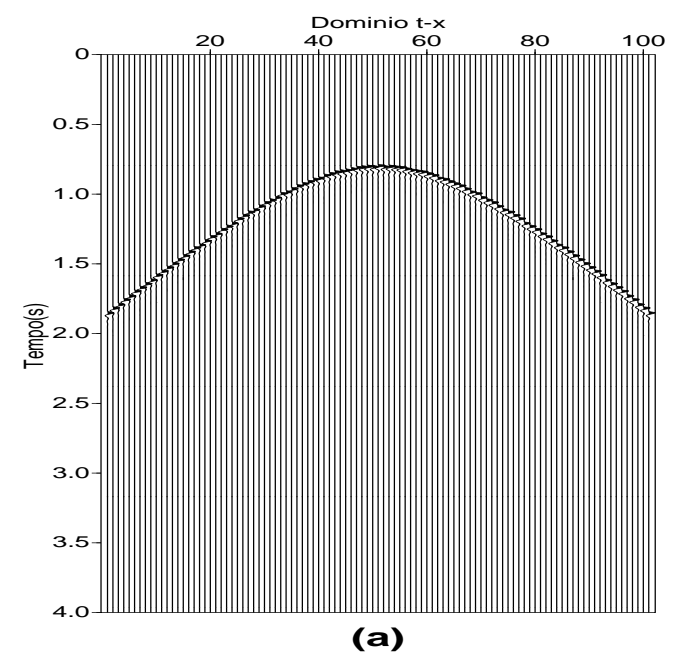

Figura 2: Curva de tempo de percurso da onda no plano $t-x$.

\section{Deconvolução de Wiener-Levinson}

A deconvolução é uma ferramenta muito eficiente para compressão do pulso sísmico e consequente aumento na resolução temporal dos eventos presentes nas seções sísmicas.

Normalmente, a deconvolução é usada antes do empilhamento. A idéia básica da deconvolução é a estimativa de um filtro inverso que quando convolvido com o pulso o converte a um impulso. Esse filtro, idealmente, deve ser capaz de restituir a resposta impulsional da Terra quando aplicado ao traço sísmico. 


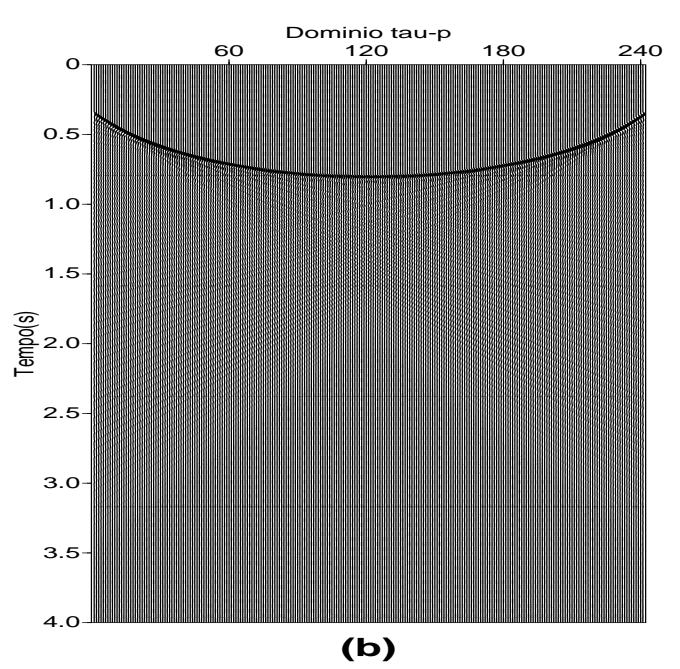

Figura 3: Curva de tempo de percurso da onda no plano $\tau-p$.

O modelo unidimensional comumente usado para representar o traço sísmico é referido como modelo convolucional, onde o traço é o resultado da convolução de um pulso gerado por uma fonte próxima a superfície com a resposta impulsional da terra acrescida do componente de ruído.

Matematicamente, o modelo convolucional do traço sísmico $x(t)$ pode ser representado por:

$$
x(t)=p(t) * e(t)+\eta(t),
$$

onde,

$x(t)$ - traço sísmico (registro do sismograma)

$p(t)$ - pulso sísmico considerado invariante no tempo

$e(t)$ - função refletividade ou a resposta impulsiva da Terra e contém, todas as reflexões primárias e ruídos

$\eta(t)$ - são os ruídos aleatórios e/ou aditivos

* - representa a operação de convolução

Nos casos em que a forma do pulso básico da fonte é conhecida, por exemplo, a assinatura da fonte, a deconvolução é considerada um problema determinístico e o filtro calculado é denominado filtro inverso. No entanto, na aquisição sísmica de reflexão a forma do pulso que chega aos diferentes geofones ou hidrofones é desconhecida, pode-se apenas controlar a assinatura do arranjo de fonte medindo-se o campo de onda em suas imediações, como normalmente ocorre em levantamentos marítimos. Por esta razão a deconvolução é realizada com base em critérios estatísticos. A teoria de predição Wiener resulta numa deconvolução estatística e o filtro estimado é chamado de operador de erro de predição, ou simplesmente, filtro de Wiener-Levinson.

O método de Wiener-Levinson consiste basicamente: (i) da estimativa dos coeficientes da função de autocorrelação do pulso, utilizando-se para tanto os traços sísmicos, (ii) da obtenção do filtro inverso, resolvendo-se as equações normais através da Recursão de Levinson, e (iii) aplicando-se o filtro inverso de WL aos traços, mediante convolução.

O processo de filtragem digital é descrito pela equação de convolução discreta, representado na forma de predição linear. A predição linear de um sinal $x_{t}$ pode ser matematicamente representada por:

$$
\tilde{x}_{t+\alpha}=\sum_{k=1}^{n} x_{t-k+1} \tilde{a}_{k}
$$

onde,

$\tilde{x}_{t+\alpha}$ - representa o sinal predito ao tempo $t+\alpha$, a partir da combinação linear dos valores de $x_{t}, \ldots, x_{t-n+1}$

$\alpha$ - representa a distância de predição

$\tilde{a}_{t}$ - é o filtro de predição

Aplicamos neste trabalho a deconvolução em sismogramas no domínio tau-p. A deconvolução empregada é a preditiva e um operador trabalha traço-a-traço, ou seja, para cada traço calculamos a função de autocorrelação e a partir desta, calculamos um filtro de deconvolução (filtro preditivo de erro) e convolvemos com os traços.

A utilização desse domínio para a atenuação do ground roll se deu em função do ground roll ser um ruído linear e normalmente aparecer em sismogramas com baixa velocidade, ou seja, alto valor de $p$. Logo, essa característica facilita a sua filtragem nesse domínio, pois o mesmo se encontra numa região diferente das reflexões, que possuem alta velocidade, isto é, valores de $p$ pequenos. Outra vantagem da filtragem no domínio $\tau-p$, é que os eventos são vistos ainda no domínio do tempo (o que possibilita uma filtragem variável no tempo), e assim a definição da região de corte para os ruídos coerentes pode ser mais facilmente escolhida.

\section{Resultados}

Desde que o objetivo deste trabalho é discutir a aplicação da deconvolução preditiva e, ao mesmo tempo, analisar qual o domínio mais adequado para sua utilização, trabalhamos inicialmente com os dados no domínio do tiro.

Para a deconvolução usamos o filtro de WL, para deconvolver os dados na presença do ground roll e luz branca de $0.1 \%$.

A figura 4(a) mostra o resultado da deconvolução preditiva aplicado num sismograma real apresentado na Figura 1.

Convertemos os sismogramas originais para o domínio tau-p e realizamos o processo de deconvolução nestes painéis. Os resultados obtidos após a deconvolução podem ser vistos e comparados àqueles obtidos com a filtragem no domínio do tiro (figura 4).

A deconvolução dos dados sísmicos no domínio taup conseguiu minimizar de maneira eficiente a influência prejudicial do ground roll, permitindo sua eliminação e possibilitando uma maior identificação das reflexões. Apesar do ruído ser o mais importante fator degradante da efetividade da deconvolução sísmica, por introduzir distorções no espectro de fase e de amplitude do sinal, conseguimos resultados satisfatórios. 
Analisando os espectros de amplitude dos dados deconvolvidos e filtrados (figura 5), observamos que o ground roll, que se encontrava presente nas baixas freqüências, foi praticamente eliminado. Nesse espectro, as reflexões apresentam um conteúdo de freqüência variando de 12 a $48 \mathrm{~Hz}$, enquanto que a alta amplitude do ground roll domina as baixas freqüências $(0 \mathrm{a} 12 \mathrm{~Hz})$. Observa-se também a presença de alta freqüência no dado devido aos efeitos da deconvolução e, em decorrência do 'aliasing' espacial causado pela transformada tau-p direta e inversa. Apesar desse problema, ainda pode-se notar uma melhora significativa da atenuação do ground roll com a utilização da deconvolução preditiva nesse domínio.

A filtragem do ground roll através de filtros de freqüência pode, em alguns casos, fornecer resultados satisfatórios. Contudo, como o ground roll é dispersivo, a filtragem atenuou bastante o ruído apesar de não apresentar uma melhor definição das reflexões. A figura 4(b) apresenta o resultado da filtragem FK aplicada sobre o mesmo sismograma de tiro comum.

A figura 6 mostra seções empilhadas dos dados. Em (a) temos o resultado da filtragem FK. Em (b) está representada a filtragem preditiva. Apesar do ruído ser o mais importante fator degradante da efetividade da deconvolução sísmica, por introduzir distorções no espectro de fase e de amplitude do sinal, conseguimos bons resultados.

\section{Conclusões}

Quando o ruído e o sinal possuem conteúdo de freqüência muito parecidos, os filtros de freqüência não são efetivos na separação dos eventos sísmicos. Contudo, o objetivo principal do método de filtragem apresentado é aumentar a resolução temporal e espacial dos dados sísmicos de maneira tal que seja possível atenuar o ground roll sem interferir no conteúdo de freqüência do sinal original.

Os métodos utilizados para a eliminação do ground roll trabalharam razoavelmente bem. No caso da filtragem de freqüência, esta foi eficiente quando o ruído não está mudando muito o seu conteúdo de freqüência, ou quando ele é bem distinto do sinal. Contudo, o filtro de freqüência pode ser muito útil nos dados em que a dispersão do ground roll não seja muito acentuada. Portanto, a sua utilização para eliminação do ground roll é justificada, apesar de não trabalhar bem em algumas situações.

Já a filtragem no domínio tau-p, possibilita uma filtragem variável no tempo, a transformação dos dados para este domínio é facilmente realizada (desde que a escolha dos parâmetros seja correta), é possível eliminar os eventos indesejáveis, sem perder informações desejáveis presentes nos dados, pois as reflexões e refrações possuem parâmetros de raio menores do que as referentes aos do ground roll, que são altos.

Portanto, a utilização da filtragem no domínio tau-p se deve ao fato desse domínio conseguir separar os eventos, e por essa razão a filtragem do ground roll pode ser mais facilmente realizada neste domínio do que nos domínios $F K$ e $t-x$, melhorando a qualidade do sinal sísmico, permitindo uma maior identificação da continuidade dos refletores.

\section{Agradecimentos}

Os autores desejam agradecer a FINEP, FAPESB e PETROBRAS pelo suporte dado ao projeto Rede em Risco Exploratório, à Paradigm e Landmark pelas licenças dos softwares de processamento sísmico doadas ao CPGGUFBA e ao PRH-08/ANP pelo financiamento na forma de bolsa de estudo dos quais tornaram possível a realização deste trabalho.

\section{Referências Bibliográficas}

Bezerra, A. C.; Porsani, M. J. (2001) Deconvolução preditiva multicanal no domínio tau-p e interpolação de traços sísmicos na atenuação de múltiplas. In: 7th International Congress of the Brazilian Geophysical Society, v.PS6, p.1221-1224, Salvador-Bahia.

Grant, F. S. and West, G. F.(1965) Interpretation theory in applied geophysics.

Lima, A. P. (1999) Deconvolução preditiva nos domínios $t-x$ e tau-p utilizando filtros Wiener-Levinson multicanais, Dissertação de Mestrado. Centro de Pós-graduação em Geofísica da Universidade Federal da Bahia.

Pestana, R. C. (1988) Deconvolução na presença de ruído coerente de alta amplitude. Tese de Doutorado. Centro de Pós-graduação em Geofísica da Universidade Federal da Bahia.

Robinson, E. A. (1984) Seismic inverson and deconvolution, Part A: Classical Methods, Geophysical Press.

Silva, M. G. (2004) Processamento de dados sísmicos da Bacia do Tacutu, Dissertação de Mestrado. Centro de Pós-graduação em Geofísica da Universidade Federal da Bahia.

Thatam, R. H. (1984) Multidimensional filtering of seismic data: Proceedings of the IEEE. In Slant-Stack processing, Society of Exploration Geophysicists, Geophysics reprint series No. 14, vol 72, no. 10. 


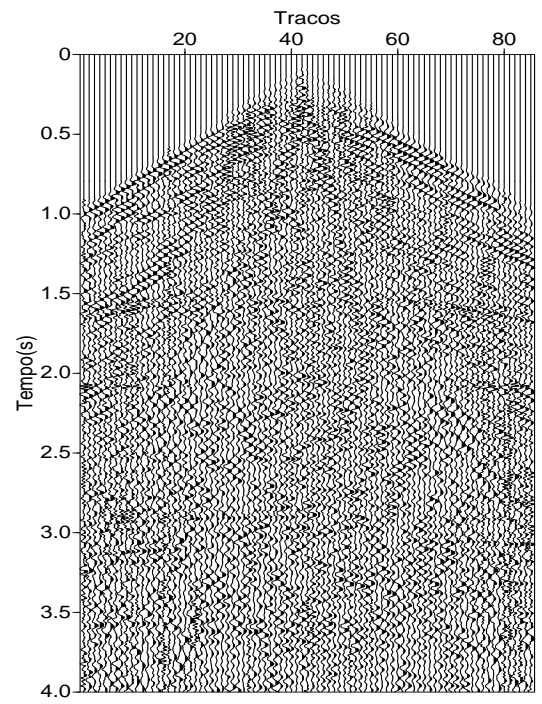

(a)

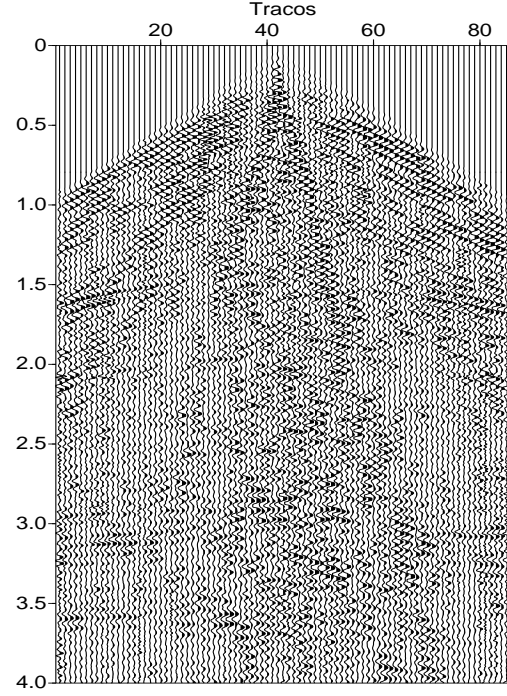

(b)

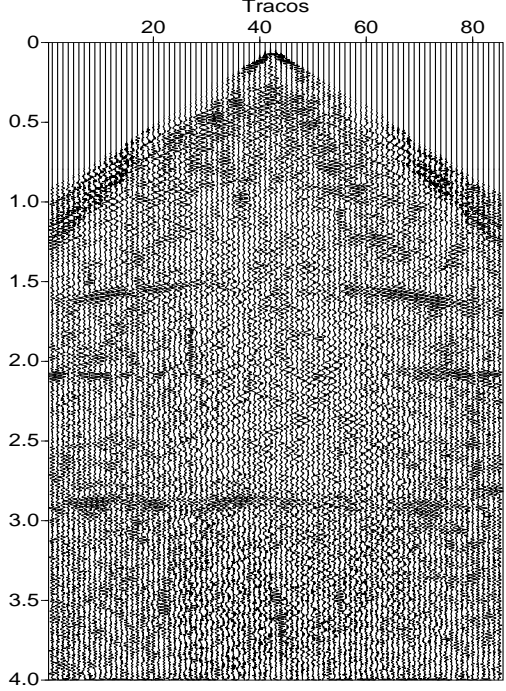

(c)

Figura 4: Resultados da atenuação do ground roll. Filtragem no domínio $t-x$ em (a). Filtragem convencional $f-k$ em (b). Filtragem no domínio $\tau-p$ em (c).

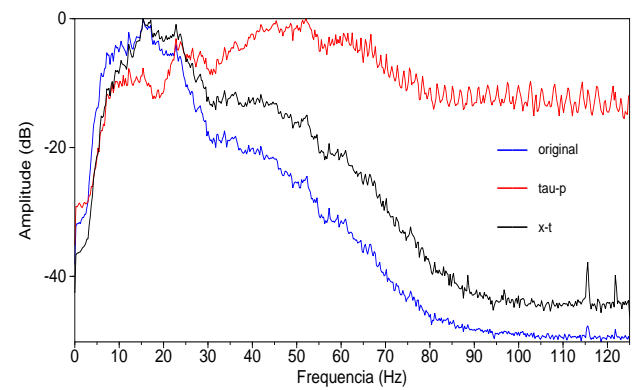

Figura 5: Espectro de amplitude. Sismograma original e após a filtragem no domínio $\tau-p$ e $t-x$.

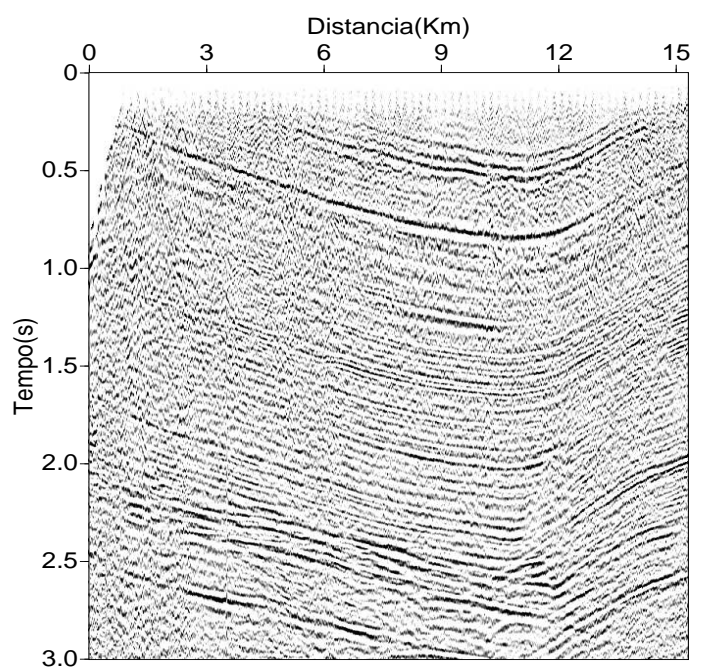

(a)

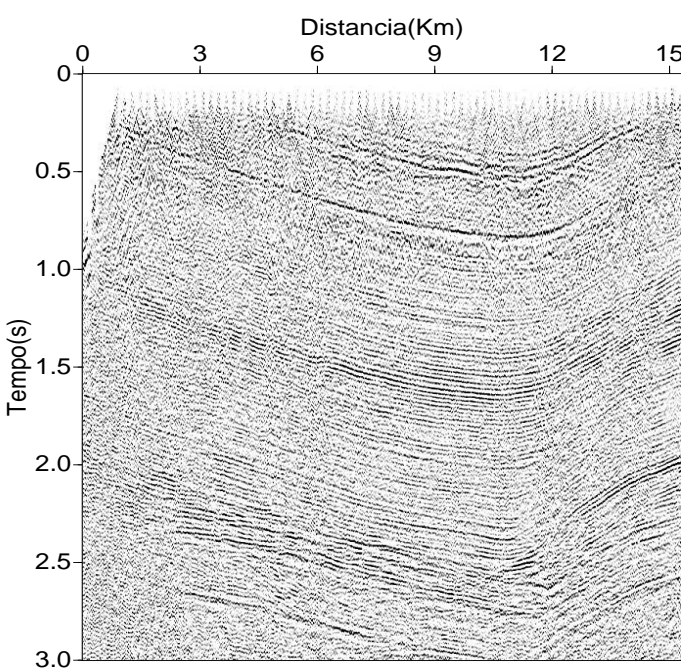

(b)

Figura 6: Seção empilhada da linha sísmica RL50-90 após filtragem do ground roll. Resultado obtido com a filtrgem FK em (a) e resultado obtido com a deconvolução WL no domínio tau-p em (b). 